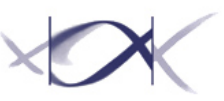

\title{
Neue Empfehlungen der Ständigen Impfkommission veröffentlicht
}

Die STIKO, die Ständige Impfkommission am Robert Koch-Institut, hat im Epidemiologischen Bulletin 34/2013 den neuen Impfkalender veröffentlicht. Hinzugekommen ist gegenüber dem Impfkalender von 2012 die Empfehlung für eine RotavirusSchutzimpfung bei Säuglingen. Veränderungen gibt es auch bei den Empfehlungen zur Hepatitis-B- und zur Influenza-Impfung.

Die neu empfohlene Rotavirus-Impfung wird als Schluckimpfung gegeben, die Impfserie sollte im Alter von sechs bis zwölf Wochen beginnen und je nach Impfstoff bis zur vollendeten 24. oder 32. Lebenswoche beendet sein. Die Impfung kann zusammen mit anderen Standardimpfungen des Säuglingsalters erfolgen. Rotaviren sind die häufigste Ursache von Magen-Darm-Infektionen bei Kindern unter fünf Jahren. Jährlich kamen bislang etwa 20.000 Kinder in Deutschland aufgrund einer Rotavirus-Infektion ins Krankenhaus. Die STIKO empfiehlt, die Impfserie frühzeitig zu beginnen. Grund ist ein möglicherweise geringfügig erhöhtes Risiko für Darminvaginationen, das mit dem Alter der Impflinge zunimmt. Unter einer Darminvagination wird die Einstülpung eines Darmabschnitts in einen anderen Abschnitt verstanden. Eine (englischsprachige) Veröffentlichung zu den wissenschaftlichen Grundlagen der Empfehlung zur Rotavirus-Impfung ist in der Juli-Ausgabe des Bundesgesundheitsblatts bereits erschienen, die deutschsprachige Begründung erscheint im Epidemiologischen Bulletin 35/2013.

Bei der Hepatitis B-Impfung hat die STIKO die Dauer des Impfschutzes mit Hilfe einer systematischen Literaturübersicht über die weltweit verfügbaren Daten bewertet. Aus den Daten wird vor dem Hintergrund der epidemiologischen Situation in Deutschland die Schlussfolgerung abgeleitet, dass nach einer in der Kindheit oder im Erwachsenenalter erfolgreich durchgeführten Grundimmunisierung im Allgemeinen keine Auffrischimpfung notwendig ist. Wie bisher wird die Kontrolle des Impferfolgs empfohlen; dabei wird untersucht, ob im Blut eine bestimmte Konzentration von Antikörpern erreicht wird. Die STIKO hat außerdem die in der bisherigen Hepatitis BImpfempfehlung aufgeführten acht Indikationsgruppen in drei Indikationsgruppen zusammengefasst (die „Standardimpfung“ gegen Hepatitis B im Säuglingsalter war nicht Gegenstand der Überarbeitung).

Bei der Influenza-Impfung empfiehlt die STIKO nun, bei Kindern im Alter von zwei bis sechs Jahren, bei denen wegen einer Grundkrankheit eine Impfung empfohlen ist, bevorzugt einen Impfstoff zu verwenden, der nicht mehr mit einer Spritze verabreicht, sondern in die Nase gesprüht wird. Damit erhofft sich die STIKO eine höhere Akzeptanz der jährlich zu wiederholenden Influenza-Impfung bei Kindern und Eltern und aufgrund der besseren Wirksamkeit eine größere Zahl vermiedener Krankheitsfälle. Im Vergleich zum Vorjahr wird die Influenza-Impfung jetzt nicht nur Personen empfohlen, die eine ungeimpfte Risikoperson betreuen, sondern auch Personen, die eine geimpfte Risikoperson betreuen. Grund ist, dass die Influenza-Impfung keinen 100\%igen Schutz bietet. Das gilt insbesondere für ältere oder immungeschwächte Menschen, die somit auch bei Impfung indirekt von einem Impfschutz der sie betreuenden Personen profitieren.

Weitere Informationen:

www.stiko.de und \& www.rki.de/impfen

\author{
Herausgeber \\ Robert Koch-Institut \\ Nordufer 20 \\ D-13353 Berlin \\ www.rki.de \\ Das Robert Koch-Institut \\ ist ein Bundesinstitut im \\ Geschäftsbereich des \\ Bundesministeriums für \\ Gesundheit \\ Pressestelle \\ Susanne Glasmacher \\ (Pressesprecherin) \\ Günther Dettweiler \\ (stellv. Pressesprecher) \\ Heidi Golisch \\ Judith Petschelt \\ Francesca Smolinski

\begin{tabular}{|c|c|}
\hline \multicolumn{2}{|c|}{ Kontakt } \\
\hline Tel.: & $\begin{array}{l}\text { O3O- } 18754-2239 \\
-2562 \text { und }-2286\end{array}$ \\
\hline $\begin{array}{l}\text { Fax: } \\
\text { E-Mail: }\end{array}$ & $\begin{array}{l}\text { O30-18754 } 2265 \\
\text { presse@rki.de }\end{array}$ \\
\hline
\end{tabular}

